ISSN: 2386-3919 - e-ISSN: 2386-3927

DOI: https://doi.org/10.14201/et20193722744

\title{
IMPORTANCIA DE LOS PRIMEROS PASOS EN LA RELACIÓN FAMILIA-COLEGIO. (DISEÑO DEL PERIODO DE ADAPTACIÓN EN EDUCACIÓN INFANTIL)
}

Importance of the first steps in the family-school relationship. (Methodological importance of the adaptation period)

\author{
Purificación CRUz CRUz \\ Facultad de Educación de Toledo. Departamento de Pedagogía \\ Universidad de Castilla la Mancha. España \\ Correo-e: Purificacion.Cruz@uclm.es \\ bttps://orcid.org/027-441-5637-3007
}

\section{Mónica Patricia BORJAS}

Universidad del Norte. Barranquilla. Colombia

Correo-e:mborjas@uninorte.edu.co

Recibido: 9/8/2019; Aceptado: 10/10/2019; Publicado: 30/12/2019

Ref. Bibl. PURIFICACIÓN CRUZ CRUZ y MÓNICA PATRICIA BORJAS. Importancia de los primeros pasos en la relación familia-colegio. (Diseño del periodo de adaptación en educación infantil). Enseñanza \& Teaching, 37, 2-2019, 27-44.

RESUMEN: La Educación es una tarea compartida entre padres y educadores (Cabrera, 2009; Zabalza, 2015). Por tanto, debemos establecer unos cauces de información y participación para establecer los primeros parámetros de actuación inicial; siendo el primer momento antes de que el alumno se incorpore al aula. Esta investigación se ha centrado en el estudio de las diferencias que presentan los niños en el periodo de adaptación, dependiendo de si han estado escolarizados previamente y el rol que han desempeñado sus familias en la crianza, con el objetivo de esclarecer la importancia que dicho periodo tiene. Se lleva a cabo una investigación aplicada "ya que se dirige a generar información objetiva sobre un problema organizativo existente en una situación real, de manera que pueda servir de apoyo a planificaciones organizativas.» (Martín-Moreno, 2007: 398), con una muestra de 238 alumnos y 15 
PURIFICACIÓN CRUZ CRUZ Y MÓNICA PATRICIA BORJAS IMPORTANCIA DE LOS PRIMEROS PASOS EN LA RELACIÓN FAMILIA-COLEGIO. (DISEÑO DEL PERIODO DE ADAPTACIÓN EN EDUCACIÓN INFANTIL)

profesionales educativos. El estudio se engloba dentro de una metodología mixta, en la que se utilizan instrumentos de corte cuantitativo (encuestas de respuesta cerrada) y cualitativo (observación participante y análisis actitudinal de los alumnos). Los resultados obtenidos demuestran que la relación existente entre las variables «adaptado de manera satisfactoria” y "escolarizado precozmente» nos hace pensar que las escuelas infantiles cumplen un papel fundamental en el desarrollo de competencias básicas necesarias para la integración del niño de tres años al nuevo centro escolar. Esto nos obliga a plantearnos la necesidad de que los docentes partan, de forma individualizada, de las habilidades, destrezas y actitudes aprendidas en la familia y/o en los centros, para fomentar, a partir de ellos, los aprendizajes escolares. Estos datos nos han ayudado a programar estrategias de actuación (Berlin, Dunning y Dodge, 2011), con el objetivo de preparar el camino para que la adaptación del niño se lleve de forma satisfactoria, sea cual sea su trayectoria anterior.

Palabras clave: familia; colegio; educación infantil; periodo de adaptación; investigación; planteamiento didáctico.

SUMMARY: Education is a task shared between parents and educators (Cabrera, 2009; Zabalza, 2015). Therefore, we must establish some channels of information and participation to establish the first parameters of initial action; being the first moment before the student joins the classroom. This research has focused on the study of the differences that children have in the adaptation period, depending on whether they have been previously educated and the role that their families have played in the upbringing, in order to clarify the importance that said period has. An applied investigation is carried out "since it is aimed at generating objective information about an existing organizational problem in a real situation, so that it can support organizational plannings (Martín-Moreno, 2007: 398), with a sample of 238 students and 15 educational professionals. The study is included in a mixed methodology, in which quantitative (closed response surveys) and qualitative (participant observation and attitudinal analysis of students) instruments are used. The results obtained show that the relationship between the variables "adapted satisfactorily» and "early schooling" makes us think that nursery schools play a fundamental role in the development of basic skills necessary for the integration of the three-year-old child in the new school center. This forces us to consider the need for teachers to start, individually, from the skills, abilities and attitudes learned in the family and / or in the centers, to encourage, from them, school learning. These data have helped us to program action strategies (Berlin, Dunning \& Dodge, 2011), with the aim of preparing the way for the child's adaptation to be carried out satisfactorily, whatever their previous trajectory is.

Key words: family; school; early childhood education; adaptation period; research; didactic approach. 
PURIFICACIÓN CRUZ CRUZ Y MÓNICA PATRICIA BORJAS

IMPORTANCIA DE LOS PRIMEROS PASOS EN LA RELACIÓN FAMILIA-COLEGIO.

(DISEÑO DEL PERIODO DE ADAPTACIÓN EN EDUCACIÓN INFANTIL)

\section{INTRODUCCIÓN}

La familia juega un papel protagonista en el desarrollo de las personas debido a que, por un lado, garantiza su supervivencia física y, por otro, realiza los aprendizajes básicos para el desenvolvimiento autónomo en sociedad: valores, lenguaje, etc. (Quintero, 2016). Cuando el niño y la niña acceden a la escuela, llegan con una historia, costumbres, aprendizajes y características que les hacen únicos, diferentes a los demás (Páez, 2015). Desde el momento en que se incorpora al centro, este se convierte en otro factor de socialización, e influirá paralelamente a la acción de los padres; de ahí que «la relación familia-escuela sea vital para su desarrollo armónico" (Vila, 2014: 12). El sentimiento de pertenencia de las familias al centro educativo, entendido como sentirse miembros y parte implicada del centro, "es una de las variables motivacionales que predispone y es consecuencia, al mismo tiempo, para la participación familiar en la escuela» (Hernández, Gomariz y Parra, 2015: p. 4). Para los padres y madres, además de conocer los objetivos educativos de la Educación Infantil y los contenidos que en ella se trabajan, es importante también que conozcan las características generales del desarrollo infantil (Aguirre, Caro, Fernández y Silvero, 2016). Ello les permitirá realizar un mejor seguimiento del proceso educativo de sus hijos/as, participar con mayor eficacia en las actividades que se realicen en el centro y una mayor comprensión de los procesos madurativos, cognitivos y de comportamiento. Este proceso también es positivo para los padres, ya que para estos también puede ser difícil la separación. Todos superan progresivamente sus miedos y ansiedades, sobre todo, si viven experiencias positivas, si la relación con el educador o la educadora es agradable, si la espera se les hace corta y, especialmente, si la separación no la viven como desaparición. "Los padres deben sensibilizarse con los cambios educativos de los hijos e identificar claramente los momentos del desarrollo en que se encuentran para apoyarlos adecuadamente» (Díaz, 2013: 46).

Según la investigación llevada a cabo por Brezinska, Mickiewicz, Czub y Ożadowiczara (2013), para algunos niños el proceso de adaptación al nuevo entorno y al nuevo rol resulta exitoso, mientras que para otros resulta complicado encontrar su lugar. El centro de Educación Infantil tiene necesariamente que organizarse de manera que los nuevos alumnos se sientan cómodos, donde el ambiente que se crea y la relación que se establezca sean básicamente afectivos. Aun así, en la escuela se vive un ritmo de vida, actividades, horarios y pautas de conductas nuevas y diferentes para los más pequeños: es un nuevo proceso de socialización (Soler, 2018). Por eso, necesitamos la colaboración e inclusión de los padres a lo largo de todo este proceso. «Posiblemente la potenciación del entorno del aprendizaje a través de los padres sea la estrategia de interrelación centro educativo-comunidad que más desarrollo haya alcanzado" (Martín-Moreno Cerillo, 2007: 236).

Para Moore (según cita Martín-Moreno, 2007), la inclusión de los padres en el entorno del aprendizaje es de importancia crítica para el rendimiento académico del alumnado por tres razones: Los padres constituyen la influencia más poderosa en la vida de los alumnos. Las escuelas deben informar a los padres sobre el currículum 
PURIFICACIÓN CRUZ CRUZ Y MÓNICA PATRICIA BORJAS IMPORTANCIA DE LOS PRIMEROS PASOS EN LA RELACIÓN FAMILIA-COLEGIO. (DISEÑO DEL PERIODO DE ADAPTACIÓN EN EDUCACIÓN INFANTIL)

de sus hijos y asesorarles para que les motiven. Y las actitudes de los alumnos hacia el centro educativo resultan influidas por los sentimientos de sus padres relativos a esta institución. Es importante que los centros educativos establezcan una buena relación con los padres y que estos perciban que el profesorado toma en cuenta su opinión cuando se analizan temas escolares. Los padres tienen percepciones directas sobre ellos que pueden ayudar al centro escolar en la organización de la necesaria individualización del entorno de aprendizaje.

1. Participación de las familias en el Periodo de AdAPtación EN El SEgundo CICLO DE EDUCACIÓN INFANTIL

\subsection{El periodo de adaptación}

Según Escolano, Bravo y Herrero (2016), el miedo a la separación parte de la idea de que el alumno no está, en principio, preparado emocionalmente para separarse del adulto del que depende, siendo su presencia tranquilizadora y su ausencia frustrante. Dado que el niño de esta edad ya es capaz de retener en la memoria objetos y personas, se alegrará al ver "caras» conocidas y, de forma complementaria, sentirá miedo ante la presencia de adultos extraños. Con todo ello, el miedo y la ansiedad que manifiestan ante la separación temporal, generalmente de los padres o adulto con quien haya establecido un vínculo afectivo básico, es uno de los principales problemas emocionales que niños y niñas afrontan.

Esta ansiedad de separación será más o menos acusada según el conocimiento que tengan el niño o la niña de la persona que se queda a su cuidado (hermano, otro familiar, canguro, maestro/a, etc.) y, sobre todo, de la calidad del vínculo que haya establecido con el adulto/s a su cuidado (los padres), siendo los miedos y ansiedades más acusados cuanto menos reforzado sea el vínculo afectivo. No es de extrañar, pues, que el niño llore desconsoladamente, incluso a veces, pierda el apetito y/o el sueño, o se muestre inactivo y apático. Por esto, si se escolariza a los niños/as tempranamente es muy importante tener en cuenta estas reacciones en ellos, y prever, durante las primeras semanas de curso, conjuntamente con los educadores, un proceso de adaptación a la escuela, el cual les ayudará a integrarse progresivamente a un nuevo ambiente, con niños y niñas de su edad, adultos que no conoce, etc. (Montalbán, 2018).

Para muchos de los niños y niñas de tres años, la asistencia al colegio supone la primera salida del ámbito familiar y, por tanto, un alejamiento de los modelos de referencia (Soler, 2018). Incluso para los que ya han asistido con anterioridad a la guardería, les exige un esfuerzo de adaptación a unos espacios, ritmos, rutinas, compañeros y adultos con los que tendrá que establecer relaciones (Muñoz, 2016). Pero, al mismo tiempo, el colegio supone una oportunidad para crecer y madurar, para afirmarse en su propia identidad e iniciar el conocimiento y la adaptación al mundo exterior. El niño y la niña tienen que ser capaces de asimilar los cambios que supone esta nueva etapa en su vida cotidiana y, para ello, el colegio debe 
PURIFICACIÓN CRUZ CRUZ Y MÓNICA PATRICIA BORJAS IMPORTANCIA DE LOS PRIMEROS PASOS EN LA RELACIÓN FAMILIA-COLEGIO. (DISEÑO DEL PERIODO DE ADAPTACIÓN EN EDUCACIÓN INFANTIL)

considerar de gran relevancia comenzar el primer curso de Educación Infantil con un periodo de adaptación (Paez, 2015).

El periodo de adaptación es «el proceso mediante el cual el niño va elaborando desde el punto de vista de los sentimientos, la pérdida y la ganancia que le supone la separación, hasta llegar a una aceptación interna de la misma” (Botella, 2018: 1). Para realizar este logro el niño y la niña necesitan, entre otras cosas, su tiempo concreto ya que la adaptación de unos y de otros será distinta (United Nations Children's Fund Education Section, 2012). Debemos conseguir, para todos nuestros alumnos, una enseñanza individualizada que les permita adaptarse a su propio ritmo, proporcionándole a cada uno las ayudas que necesite según sus características, siempre teniendo en cuenta sus destrezas, carencias, historia y emociones (Garreta, 2015). Por tanto, el primer paso, por parte del docente y con la colaboración de las familias, es descubrir la historia previa a la incorporación del alumno al centro, su historial académico, emocional y familiar y así poder planificar la actuación individualizada que abarque a toda la comunidad educativa (Petrakos y Lehrer, 2011). El segundo paso sería la creación del rol participante de las familias.

\subsection{Participación de las familias}

Convencidos como estamos de la importancia de dicha participación, es inquietante comprobar la escasa investigación que existe sobre este tema y especialmente la nula aportación metodológica sobre la buena praxis del establecimiento de relación familia-escuela en este periodo crítico. Espiñeira (2017) analiza la participación de las familias en el periodo de adaptación, llevando a cabo una revisión legislativa y bibliográfica. Mostrando los siguientes resultados según los descriptores utilizados: Adaptación + escuela infantil: 30. Adaptación + educación infantil: 93. Periodo de adaptación: 316. Planificación del periodo de adaptación: 5. Plan de periodo de adaptación: 10. Participación familias + periodo de adaptación: 0. Colaboración familias + periodo de adaptación: 2. Orientación familias + periodo de adaptación: 2. Participación familias + escuela infantil: 20. Colaboración familias + escuela infantil: 9. Orientación familias + escuela infantil: 4. Participación familias + educación infantil: 33. Colaboración familias + educación infantil: 18. Orientación familias + educación infantil: 8. Total: 550 (Espiñeira, 2017: 273-277). Los documentos donde se trata de forma específica los descriptores familia-periodo de adaptación suman un total de 96 frente a los 454 restantes. Como puede comprobarse, el 37.71\% de los documentos investigados, según la autora, incluyen el descriptor "periodo de adaptación", pero en ninguno de ellos se estudia o menciona la concreción de la participación de las familias. Siendo significativo ya que numerosos estudios afirman la importancia de dicha relación en este periodo. Lo que probablemente falta a los educadores son vías concretas para articular esa colaboración y formas de acción que vayan gradualmente salvando la separación que actualmente marca las relaciones con los padres. Como ocurre en otros ámbitos, en este el cambio de 
PURIFICACIÓN CRUZ CRUZ Y MÓNICA PATRICIA BORJAS IMPORTANCIA DE LOS PRIMEROS PASOS EN LA RELACIÓN FAMILIA-COLEGIO. (DISEÑO DEL PERIODO DE ADAPTACIÓN EN EDUCACIÓN INFANTIL)

mentalidad y el cambio en la práctica ocurrirán simultáneamente e irán el uno de la mano del otro (Martín, 2015).

\subsection{Construcción del rol participante}

La participación de los padres del alumnado en los centros escolares es un principio reconocido en la Constitución española, en su artículo 27,7, estableciendo la intervención de los padres en el control y gestión de los centros. Entrando en la legislación específica del sistema educativo, se observa que la participación de la comunidad es un principio constante en las leyes actualmente en vigor. Representantes de los padres del alumnado y de la comunidad forman parte del consejo escolar, órgano donde participan en las tomas de decisiones institucionales. Pudiendo participar, también, a través de las asociaciones, federaciones, grupos de trabajo, comisiones, etc. Y de forma más particular su participación puede llevarse a cabo a través de tutorías, reuniones, talleres, seminarios y escuelas de padres. Para alcanzar estos objetivos, Greene y Habana-Hafner (2003) opinan que es preciso crear una cultura escolar abierta y de cooperación que disponga de vías eficaces para que los padres puedan plantear preguntas, exponer preocupaciones y/o sugerencias (según cita Martín-Moreno 2007: 238). Una forma de conceptualizar esa cultura integradora, es distinguir entre la implicación esporádica y la implicación sistemática. La primera es la que ocurre en determinados momentos especiales que se caracterizan, entre otras cosas, por su frecuencia baja y/o asistemática: colaboración en el periodo de adaptación, participación en salidas extraescolares especiales o en fiestas que se organicen en la Escuela Infantil, ayuda en la preparación de materiales para el trabajo con los niños, etc. La implicación sistemática es aquella que tiene un carácter más regular, más estable y que forma parte de la metodología de trabajo con los niños y niñas: asistencia regular y organizada de padres en el aula, colaboración regular y planificada en el trabajo educativo, participación en determinados talleres o actividades regulares, trabajo por comunidades de aprendizaje, etc.

\subsection{Periodo de adaptación y familia}

La implicación de los padres durante el periodo de adaptación es una de las actividades de cooperación más importantes por las repercusiones que tiene en el bienestar del niño y su vivencia de la escuela. Tratándose de niños pequeños, acostumbrarse a la separación no solo resulta difícil para ellos, sino también para sus padres, cuya tranquilidad durante el proceso de adaptación va a ser decisiva (Julio, Girón y Navarro, 2012).

Hay que tomar en consideración distintas estrategias para asegurar que el objetivo de facilitar la adaptación se cumpla: conseguir un clima de tranquilidad, de ahí la necesidad de contar con la presencia, durante los primeros días, de algún familiar y darles pautas sobre qué hacer mientras están presentes; permitir al niño explorar un nuevo espacio, conocer caras nuevas, hacerse a la relación con adultos 
PURIFICACIÓN CRUZ CRUZ Y MÓNICA PATRICIA BORJAS IMPORTANCIA DE LOS PRIMEROS PASOS EN LA RELACIÓN FAMILIA-COLEGIO. (DISEÑO DEL PERIODO DE ADAPTACIÓN EN EDUCACIÓN INFANTIL)

desconocidos, etc., desde la seguridad que transmite la presencia de su figura de apego; se propondrán varias actividades en el aula para que los padres puedan integrarse en el ambiente del colegio (León, 2011; Monarca, 2013). Actividades del tipo cuentacuentos, meriendas compartidas, creación del álbum de la clase, representación de títeres..., y, por último, la información centro-familia, familia-centro deberá ser continua, fluida, cordial y tranquilizadora.

El primer contacto, según Sánchez (2011), que debe producirse mucho antes de que comience el curso escolar, debe tener el objetivo de informar sobre la importancia de este ciclo, haciéndoles ver su carácter educativo y no solo asistencial, los aspectos fundamentales para su buen desarrollo y la organización del periodo de adaptación (no olvidemos que los padres deben reorganizar su horario laboral para adaptarlo a las necesidades psicopedagógicas de ampliación de horario de los primeros días).

Se ha observado que los niños difieren mucho unos de otros en su proceso adaptativo (Chacón, 2015). Este proceso, muy complejo y que vemos repetidamente año tras año al comenzar el curso escolar, hace que nos detengamos para preguntarnos: ¿Cómo influye el que un niño haya estado escolarizado antes, en el proceso de adaptación al ingresar en Educación Infantil? ¿Sufren más los niños que vienen de casa? ¿Depende ese desarrollo de la forma de educar en la familia? ¿Cómo afecta al niño la primera separación de sus padres o familiar que le cuida? ¿Cómo podríamos hacer para que padres y centro consigan mayor coordinación y un proyecto en común para facilitar el éxito en el proceso?

Queremos conseguir para todos nuestros alumnos una enseñanza individualizada, que respete los ritmos de aprendizaje de cada niño, proporcionando a cada uno de ellos, las herramientas que necesiten según sus características. Es de suma importancia conocer sus circunstancias y sus respectivos ambientes familiares para plantear un trabajo metodológico de calidad que ayude a la toma de conciencia sobre los roles que ejercen los padres en relación a la "crianza» de sus hijos, y la importancia de un ambiente familiar estimulante. Cabe mencionar el rol que deberá cumplir el educador no solo como guía y orientador de los niños, sino también de las familias (Pérez, 2011).

\section{Metodología}

El objetivo principal que se persigue es analizar una de las posibles causas de la exitosa adaptación del niño al centro educativo y su origen en el rol que desempeñan las familias en la crianza 0-3 años. Quedando planteada la hipótesis de la siguiente manera: Los niños que vienen de otros centros infantiles o guarderías ya han pasado por un periodo de adaptación y, por lo tanto, son más flexibles a la hora de adaptarse a un nuevo colegio. Se espera que los resultados proporcionen un enfoque real de la percepción sobre adaptación infantil y estimulación familiar y, de paso, dar respuesta a las interrogantes que se formulan los docentes de Educación Infantil cada vez que se tienen que enfrentar al inicio de un nuevo curso. 
Se lleva a cabo una investigación aplicada, «ya que se dirige a generar información objetiva sobre un problema organizativo existente en una situación real, de manera que pueda servir de apoyo a planificaciones organizativas.» (Martín-Moreno 2007: 398). El estudio se engloba dentro de una metodología mixta, en la que se utilizan instrumentos de corte cuantitativo (encuestas) y cualitativo (observación participante y análisis actitudinales de los alumnos). El objetivo general de la investigación es comprobar si existe diferencia entre los niños que vienen de casa y los que vienen de otros centros y así poder tomar las medidas pedagógicas pertinentes. Los objetivos específicos quedan establecidos como:

- Diferenciar rasgos conductuales de niños criados en diferentes situaciones.

- Proponer nuevas estrategias metodológicas, aplicables a los padres y docentes, en el proceso de adaptación.

- Integrar a los padres en las actividades durante las primeras semanas.

\subsection{Participantes}

La muestra seleccionada, por conveniencia, la compone un total de 238 alumnos de 2-3 años que inician su escolarización en el segundo ciclo de Educación Infantil y las 10 tutoras-maestras. La selección, no aleatoria, se llevó a cabo durante las primeras semanas del comienzo del curso español, es decir, durante el periodo de adaptación. Esta muestra la componen 10 grupos del primer nivel del segundo Ciclo de Educación Infantil de 7 centros de la provincia de Toledo $(5$ centros públicos y 2 centros concertados). Dentro de cada aula hay un número no preestablecido de niños que proceden de guardería y niños que han estado en casa. La edad varía en meses de unos a otros, pero todos los miembros cumplen 3 años antes del mes de diciembre (importante para controlar la variable extraña: "madurez biológica»). Se ha considerado el procedimiento ético y confidencialidad de recogida de datos y los permisos pertinentes de las familias y de los centros para la aplicación de los instrumentos.

\subsection{Instrumentos de evaluación}

Se han utilizado distintos instrumentos de evaluación: En primer lugar, se ha realizado un análisis cualitativo de los datos generales de cada uno de los alumnos (datos generales y familiares, datos de escolarización previa, salud, conducta emocional-social, hábitos, juegos, relaciones y adaptación al centro), utilizando el cuestionario aportado por la editorial Algaida (recuperado de http://cort.as/-MQRj), cumplimentado por las familias antes de iniciarse el periodo de adaptación y la profesora una vez finalizado el periodo de adaptación. Otra fuente utilizada ha sido la observación participante como forma eficaz de conocer paso a paso todo el proceso de adaptación en niños tan pequeños, obteniendo datos cualitativos que los empíricos no podrían facilitarnos y conservando el rigor científico en su recogida. Pidiendo a cada una de las tutoras que utilizaran un anecdotario o relato 
PURIFICACIÓN CRUZ CRUZ Y MÓNICA PATRICIA BORJAS IMPORTANCIA DE LOS PRIMEROS PASOS EN LA RELACIÓN FAMILIA-COLEGIO. (DISEÑO DEL PERIODO DE ADAPTACIÓN EN EDUCACIÓN INFANTIL)

descriptivo de episodios o anécdotas significativas, vinculadas con un aspecto de la conducta del sujeto observado. Por último, se ha elaborado y validado (a través de la intervención de cuatro maestras especialistas, una psicopedagoga, el orientador de uno de los centros participantes y una prueba piloto, en una clase de 18 niños/ niñas de tres años, en otro centro fuera de la muestra) un cuestionario, donde se ha negociado con el grupo de expertos los temas o variables importantes sobre los que focalizar la atención en la recogida de datos y poder categorizarlos. Las categorías descriptivas, sobre las que posteriormente se discutieron los resultados, quedaron establecidas en:

- Agrado o no en la asistencia a clase: se entiende que asisten a clase con más agrado aquellos que se presentan con menor número de conductas negativas hacia la misma, entendiendo como tales: no querer ir, excusas no justificadas, llantos y rabietas, intentos de escapar de clase, etc.

- Independencia y autonomía personal: hábitos, destrezas, capacidades y habilidades propias de esta edad de forma independiente o apoyado por el adulto.

- Relaciones con los demás: adultos y compañeros.

El cuestionario definitivo incluía las instrucciones básicas para las docentes (cómo rellenar cada una de las cuestiones, temporalización de la aplicación una vez finalizado el periodo de adaptación y utilización de sus datos de observación para incluirlos en cada una de las categorías definidas) y dos apartados a cumplimentar, el primero con los datos generales del alumno y el segundo dirigido directamente al análisis de las tres variables establecidas, dando una puntuación de 0 a 5 en cada uno de los apartados (marcando el 0 en actitudes más disruptivas y el 5 en actitudes más positivas). La puntuación de cada alumno es la suma de las respuestas al cuestionario, estableciendo un límite de puntuación para la asignación de los sujetos a cada uno de los grupos que se formarán con posterioridad. En un primer momento, solo nos interesa la frecuencia de sujetos que pertenecen a cada grupo, aunque la puntuación personal se utilizaría, con posterioridad, para la evaluación individual y el estudio de otras características de interés.

\subsection{Procedimiento}

Una vez definido el problema y planteados los objetivos, se establece y realiza el siguiente proceso en la investigación:

- Antes del inicio del periodo de adaptación: Análisis, por parte de las investigadoras, del cuestionario cumplimentado por los padres en los primeros días de clase. Realización de tablas de frecuencias de niños de un grupo (sí escolarizados previamente) y de otro grupo (no escolarizado previamente). Definición de la temporalización del proceso. 
- Durante el periodo de adaptación: Análisis de aspectos de interés destacados en los anecdotarios elaborados por las tutoras de los diez grupos de alumnos participantes. Entrevista con las tutoras para explicar el proceso de recogida de datos: se les expone las normas a tener en cuenta para rellenar los cuestionarios y se resuelven las dudas de su aplicación.

- Después del periodo de adaptación: Análisis de los cuestionarios. Reunión de las docentes, coordinadoras, compañeros profesionales e investigadoras, para, una vez analizada toda la información, asignar a cada uno de los alumnos a los siguientes grupos: niños sin ningún problema de adaptación, niños con leves problemas de adaptación y niños con significativos problemas de adaptación. Para la asignación de cada uno de los sujetos se puntuaron con un 1 si la respuesta es sí y un 0 si la respuesta es no. Realización de tabla de frecuencia de alumnos según su adaptación al centro. Análisis estadístico para comprobar la posible relación entre las dos variables.

\subsection{Análisis}

Se ha estimado que en modo alguno podía entenderse ni un nivel de medición de intervalos ni que los supuestos de las pruebas paramétricas se cumplían, razón por la cual se pensó en una prueba no paramétrica. La medición nominal que se ha llevado a cabo, a través de los cuestionarios, da lugar a la asignación de los sujetos a las categorías en que se ha dividido el conjunto, calculando las frecuencias de cada celdilla. Los datos obtenidos han sido: Niños escolarizados previamente: 154; Niños no escolarizados previamente: 82; Total de niños escolarizados: 236; Niños sin ningún problema de adaptación: 82; Niños con leves problemas de adaptación: 72; Niños con significativos problemas de adaptación: 82.

A continuación se muestra la siguiente tabla bidimensional que nos permitirá establecer determinadas asociaciones entre las frecuencias tratadas:

\section{TABLA 1}

Tabla bidimensional con asociaciones de frecuencias

\begin{tabular}{ccccc}
\hline & $\begin{array}{c}\text { Niños sin ningún } \\
\text { problema de } \\
\text { adaptación }\end{array}$ & $\begin{array}{c}\text { Niños con leves } \\
\text { problemas de } \\
\text { adaptación }\end{array}$ & $\begin{array}{c}\text { Niños con significativos } \\
\text { problemas de adaptación }\end{array}$ & Total \\
\hline Previamente & 28,49 & 25,05 & 28,49 & 154 \\
escolarizados & 77 & 46 & 31 & 82 \\
\hline No escolarizados & 53,51 & 46,98 & 53,51 & 236 \\
\hline Total & 5 & 26 & 82 & 236 \\
\hline
\end{tabular}

Elaboración propia. 
Las frecuencias esperadas en cada celdilla no son demasiado pequeñas, y son mayores de 1 en más del 20 por ciento de las casillas. Observando la tasa de frecuencias de niños que no presentaban problemas, se llega a la conclusión de que los niños escolarizados previamente consiguen una mayor adaptación al centro o, por lo menos, con inferior dificultad, que los compañeros que no han asistido a un centro o guardería previamente.

\section{GRÁFICO 1}

Gráfico de asociaciones de frecuencias

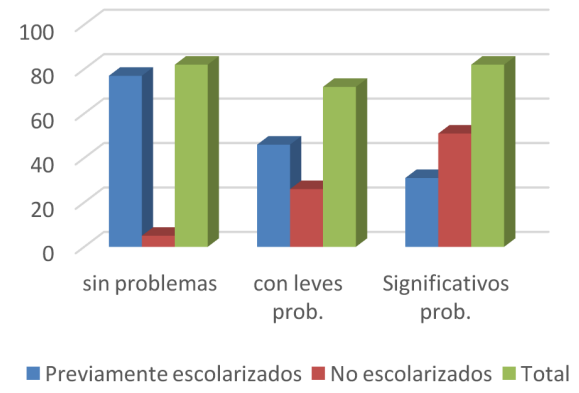

Elaboración propia.

Por tanto, rechazamos la Ho que postulaba la no diferencia de los niños que provenían de guardería de los que venían de estar asistidos en casa, ante el momento de enfrentarse a la adaptación al centro de E. Infantil, con un nivel de confianza de 0,99 (99\%). Aunque no podemos obviar que esta investigación puede estar sesgada por el distinto estilo educativo de las profesoras; por la maduración de los alumnos más pequeños de cada grupo, no es lo mismo el desarrollo madurativo de un niño nacido en el mes de enero que en el mes de diciembre, y por la falta de generalización de los resultados, ya que el estudio solo se ha realizado en la provincia de Toledo. No obstante, conviene resaltar que el origen de la investigación está generalizado en la población infantil ya que las características psicoevolutivas de los niños de esta edad son muy parecidas, siempre que su situación social-familiar tenga similares características. Para considerar una generalización a la población debería llevarse a cabo una investigación con una muestra aleatoria simple o estratificada. Proceso que se está iniciando en la ciudad de Barranquilla en colaboración con la Universidad del Norte.

\section{DisCUSIÓN}

Al realizar una comparación de las diferentes situaciones y vivencias de los alumnos, centro de la investigación, se pudo constatar la diferencia conductual de los niños criados en diferentes situaciones. Se concluye así que, para los niños de $2 .^{\circ}$ 
PURIFICACIÓN CRUZ CRUZ Y MÓNICA PATRICIA BORJAS IMPORTANCIA DE LOS PRIMEROS PASOS EN LA RELACIÓN FAMILIA-COLEGIO. (DISEÑO DEL PERIODO DE ADAPTACIÓN EN EDUCACIÓN INFANTIL)

ciclo de Educación Infantil, el haber estado ya escolarizados puede influir de forma positiva en el proceso de adaptación a un nuevo centro. Como ya anticipábamos al inicio de este artículo, la relación existente entre las variables «adaptado de manera satisfactoria» $\mathrm{y}$ "escolarizado precozmente» nos hace pensar que las escuelas infantiles cumplen un papel fundamental en el desarrollo de competencias básicas necesarias para la integración del niño de tres años al nuevo centro escolar. No discutimos, en ningún momento, que el lugar por excelencia para cuidar al niño sea su familia y su casa, pero debemos reconocer que una serie de aspectos quedan un tanto descuidados o no desarrollados y que hacen que nuestros hijos «sufran» un poco más al integrarse en el ambiente escolar. Como educadoras de E. Infantil, debemos desempeñar nuestro rol, además de proyectar nuestras habilidades y conocimientos a la comunidad y de forma especial a los padres (Garreta, 2015).

A partir de este análisis y teniendo como base las múltiples investigaciones que afirman la importancia de la colaboración familia-centro, «resulta esencial ser conscientes, que si ambas estructuras deben y desean trabajar a la par, construir un proyecto común representa una de las primeras empresas en las que invertir nuestros esfuerzos» (Esteban, 2015). En primer lugar, se aprecia la necesidad de que los docentes partan de las habilidades, destrezas y actitudes aprendidas en la familia, para fomentar, a partir de ellos, los aprendizajes escolares. De esta forma la enseñanza se contextualiza y se refiere a la individualidad del sujeto, teniendo también una función compensadora de las desigualdades que puedan producirse en el entorno familiar o seguidora de las buenas praxis recibidas en su crianza. Esta idea es apoyada por el MECD, destacando la existencia de «una amplia evidencia empírica que indica que la participación de las familias en la escuela, además de constituir un derecho y un deber, aporta grandes beneficios, tanto a los estudiantes como a la escuela y a los propios padres y madres» (Vallespir, Rincón y Morey, 2016: 32).

En un análisis pormenorizado de cada una de las variables, se llegó a la conclusión de que los niños que no asisten a centros asistenciales tienen carencias en aspectos fundamentales como el desarrollo de autonomía personal (pedir ayuda, intentar hacer las cosas por sí solos, etc.) y, sobre todo, cuentan con menos hábitos adquiridos (aseo personal, control de esfínteres, recoger, etc.). También es evidente que estos niños tienen más dificultades para separarse de su familia y relacionarse con otros adultos y niños de su edad. Estamos de acuerdo con las conclusiones aportadas por United Nations Children's Fund Education Section, que indica que las tres dimensiones de la preparación escolar son: Niños preparados, centrados en el aprendizaje y el desarrollo de los niños. Preparar las escuelas, centrándose en el entorno escolar junto con las prácticas que fomentan y apoyan una transición sin problemas para los niños. Preparar a las familias, centrándose en las actitudes de los padres y cuidadores y la participación en el aprendizaje y desarrollo tempranos de sus hijos y la transición a la escuela (2012).

Así mismo y de acuerdo con los resultados obtenidos por otros autores, esta investigación ha intentado mostrar el importante papel de los factores subjetivos: la edad y el nivel de desarrollo general de un niño en el proceso de adaptación; 
PURIFICACIÓN CRUZ CRUZ Y MÓNICA PATRICIA BORJAS IMPORTANCIA DE LOS PRIMEROS PASOS EN LA RELACIÓN FAMILIA-COLEGIO.

(DISEÑO DEL PERIODO DE ADAPTACIÓN EN EDUCACIÓN INFANTIL)

conocimiento de experiencias previas; la ayuda familiar para que sea reconfortante la transición, y la programación anticipada, justificada e intencionada del periodo de adaptación por parte de la comunidad educativa (Poole, Miller y Booth, 2019). Sabemos de las carencias que esta investigación presenta, pero queríamos buscar el punto de partida para hacer entender a las familias, equipos directivos, comunidad educativa e incluso las entidades políticas, la importancia del periodo de adaptación en el segundo ciclo de Educación Infantil, la importancia del sacrificio horario que este supone y la importancia de una buena programación y praxis a lo largo del proceso.

\section{CONCLUSIÓN E IMPLICACIONES PARA LA PRÁCTICA DOCENTE}

Aún quedan por abordar los dos últimos objetivos específicos planteados en el inicio de la investigación: Proponer nuevas estrategias metodológicas, aplicables a los padres y docentes, en el proceso de adaptación e integrar a los padres en las actividades, durante las primeras semanas. Aspectos fundamentales que tienen que redirigir la práctica metodológica del docente. Y fue en la última reunión, donde se compartieron todos los resultados de la investigación, cuando el grupo de profesoras y profesionales tomó una serie de decisiones para mejorar el periodo de adaptación en sus respectivos centros, siempre respetando la normativa legislativa vigente, planificando un proyecto de actividades para realizar en coordinación con las familias y compensar esos aspectos carenciales que se habían observado y analizado (Blanco, 2014).

\subsection{Proyecto de trabajo centro-familia para abordar el periodo de adaptación:}

Objetivos:

- Lograr un acercamiento y participación entre todas las partes involucradas: niños, padres, familias y educadores.

- Hacer que la inserción del niño se produzca de forma eficaz.

- Crear lazos afectivos.

- Ser «receptores» de las necesidades, demandas, intereses e inquietudes de cada niño, de cada niña y de su familia.

Contenidos:

- Acercamiento e interrelación entre las partes implicadas en el proceso de incorporación.

- Conocimiento del centro y sus materiales.

- Invitación a las familias a compartir diversas actividades.

- Trabajo de hábitos, destrezas y actitudes desde una metodología individualizada.

- Creación del rol participante de las familias. 
Propuestas pedagógicas e instrumentales para este periodo:

- Es fundamental explicar a las familias en qué consiste el periodo de adaptación y su justificación, buscando su colaboración y confianza en que este proceso se llevará a cabo de la mejor manera posible. Por eso, se tendrá una reunión a final de junio para dar a conocer a los padres, la información necesaria y los puntos de colaboración a trabajar en el verano. Y una segunda reunión durante el periodo de adaptación.

- Para un mejor conocimiento de los procesos de cada niño, es aconsejable recibir a grupos pequeños que se vayan conociendo entre sí y reconociendo su espacio y a su tutor/a. Es importante programar los periodos de permanencia en el centro que deberán ir aumentando poco a poco y progresivamente para afianzar la seguridad y confianza del niño.

- La distribución de los niños en las aulas se hará en relación a los datos obtenidos de la encuesta que los padres han cumplimentado al entregar la matrícula, en el mes de julio. Es necesario contar con la mayor información posible sobre cada alumno y su entorno familiar. Para ello, se tendrá una entrevista individual con cada familia. Esto se llevará a cabo en los primeros días. Y se les pedirá a los padres que rellenen un cuestionario sobre el desarrollo madurativo de su hijo/a.

- Se ofrecerán unos consejos que faciliten el proceso: Desdramatizar la incorporación del niño/a al centro de Educación Infantil. Conocer el/la maestro/a de su hijo/a. Conocer el aula de su hijo/a y algunas dependencias del centro. Conocer los objetivos de la Educación Infantil y la dinámica a seguir durante el curso. Colaborar en el periodo de adaptación. Contarle aspectos positivos: que tiene un patio grande para jugar, una clase bonita, cuentos, pinturas, plastilina, amigos nuevos... Darle seguridad y confianza, sobre todo, en los primeros días. Acompañarle hasta su sitio de recogida asignado. Hablar con el niño/a cuando llega del colegio y preguntarle qué hizo, qué le gusto más, qué le gustó menos... Estar en la puerta del colegio a la hora de salida con extrema puntualidad. No sobreprotegerlo en exceso. Darle autonomía en las rutinas diarias de casa. Confiar siempre en la profesionalidad del tutor/a (Valles, 2014).

- Se propondrán varias actividades en el aula para que los padres puedan integrarse en el ambiente del colegio (Cano, 2015). Actividades del tipo cuentacuentos, meriendas compartidas, creación del álbum de la clase, representación de títeres, etc.

- La información centro-familia, familia-centro deberá ser continua y fluida, especialmente en este periodo. Las tutoras informarán diariamente a los papás de los pormenores del día o posibles incidencias. Esta comunicación será en un tono cordial y tranquilizador. 
PURIFICACIÓN CRUZ CRUZ Y MÓNICA PATRICIA BORJAS IMPORTANCIA DE LOS PRIMEROS PASOS EN LA RELACIÓN FAMILIA-COLEGIO. (DISEÑO DEL PERIODO DE ADAPTACIÓN EN EDUCACIÓN INFANTIL)

- Es primordial que los padres colaboren en la adaptación de su hijo (MartínQuintana, 2009) y para conseguir esto es importante que el niño esté acostumbrado a realizar una serie de tareas en casa que impliquen una autonomía e inteligencia emocional que evite diferencias entre la casa y la escuela, y que apoye la labor del docente. Por esta razón a los padres se les invita a realizar, en casa, las siguientes actividades y así potenciar las diferentes áreas de desarrollo de su hijo:

- Área del Lenguaje: Hablar con el niño/a cuando llega del colegio y preguntarle qué hizo, qué le gustó más... Expresar qué siente emocionalmente tanto de forma oral como gestual. Reforzar la dicción correcta de fonemas y ayudarles a construir frases gramaticalmente más complejas.

- Área de Hábitos y Autonomía: Vestirse y desvestirse, primero con ayuda para después hacerlo solo. Controlar esfínteres e ir al baño de forma autónoma. Eliminar el pañal de la noche si no existe un problema de enuresis. Aprender a limpiarse sin necesidad de un adulto. Eliminar biberones y chupetes que pueden producir malformaciones dentales y retraso en el lenguaje oral. Enseñarle a tener una buena limpieza bucal. Tener una buena rutina de descanso.

- Juego: Proporcionarle situaciones de juego con sus iguales, con adultos y en solitario.

- Evitar la sobreprotección. Mantener normas, rutinas y responsabilidades dentro del entorno familiar.

- Fomentar la tolerancia a la frustración.

El proceso se da por terminado cuando el niño y la niña se muestran contentos, cuando hablan e intercambian experiencias, cuando utilizan los objetos de la clase, cuando se mueven con seguridad y naturalidad y cuando su rostro muestra una sonrisa al entrar o salir del aula... y la «seño» le recibe o despide con una sonrisa... y su mamá o papá le despide o recibe con una sonrisa, mostrando seguridad y confianza.

Esta investigación solo es un pequeño detalle de lo mucho que queda por hacer en el mundo educativo infantil. Aún queda seguir investigando sobre el beneficio de la intervención de las familias en los centros, sobre la poca participación de estos, sobre la relación entre participación y situación social-económica y sobre los aspectos, según las maestras que han intervenido en el estudio, que hacen que los niños muestren más problemas de adaptación a contextos educativos. Pero principalmente debemos seguir trabajando para empoderar este ciclo y resaltar su valor más allá del asistencial. Suerte que los últimos estudios de la neurociencia empiezan a cumplir esa labor. 
PURIFICACIÓN CRUZ CRUZ Y MÓNICA PATRICIA BORJAS

IMPORTANCIA DE LOS PRIMEROS PASOS EN LA RELACIÓN FAMILIA-COLEGIO.

(DISEÑO DEL PERIODO DE ADAPTACIÓN EN EDUCACIÓN INFANTIL)

\section{REFERENCIAS BIBLIOGRÁFICAS}

Aguirre, A.; Caro, C.; Fernández, S. y Silvero, M. (2016). Familia, escuela y sociedad. Manual para maestros. Recuperado de http://cort.as/-MQoU.

Algaida (2012). Cuestionario inicial. Papelillos 3 años. Recuperado de http://cort.as/-MQRj

Ballester, LL.; Nadal, A. y Amer, J. (2017). Métodos y técnicas de investigación educativa. Mallorca: Universitat de les Illes Balears.

Berlin, L. J.; Dunning, R. D. y Dodge, K. A. (2011). Enhancing the transition to kindergarten: A randomized trial to test the efficacy on the "stars" summer kindergarten orientation program. Early Childhood Research Quarterly, 26, 124-139. Durhan: Center For Child and Family Policy Duke University.

Blanco Blanco, M. ${ }^{a}$ V. (2014). Una propuesta de trabajo para implicar a las familias en las escuelas de Educación Infantil. Trabajo Fin de Grado. Universidad de Cádiz. Recuperado de https://n9.cl/zBld.

Botella, R. (2018). El periodo de adaptación. Recuperado de https://n9.cl/Uu5h.

Brezinska, A. I.; Czub M. y Ożadowicz N. (2013). Adaptation of three-year-old child to preschool environment. Edukacja. An interdisciplinary approach, 1, 25-40. Poznan. ISSN 0239-6858.

Cabrera, M. (2009). La importancia de la colaboración familia-escuela en la educación. Recuperado de https://n9.cl/vVL4.

Cano, R. y Casado, M. (2015). Escuela y familia. Dos pilares fundamentales para unas buenas prácticas de orientación a través de escuelas de padres. Revista Electrónica Interuniversitaria de Formación del Profesorado (REIFOP), 18 (2), 15-27.

Chacón-Ortiz, M. (2015). El proceso de evaluación en educación no formal: un camino para su construcción. Revista Electrónica Educare, 19 (2), 21-35. Recuperado de http://cort. as/-MQoa.

Consejo Escolar del Estado (2015). La participación de las familias en la educación escolar. Madrid: Ministerio de Educación, Cultura y Deporte. Recuperado de http://xurl.es/dot4́b.

Díaz, A. (2013). Acompañamiento de los padres en la tarea educativa de sus hijos/as y su incidencia en el aprendizaje de los niños/as del $1^{\circ}$ y $2^{\circ}$ ciclos. (Tesis de Licenciatura) Recuperado de https://n9.cl/y8hV.

Escolano, E.; Bravo, M. y Herrero, M. A. (2016). Optimización del desarrollo y aprendizaje infantil a través del fomento de apego seguro en los maestros. En Inteligencia Emocional y Bienestar II: reflexiones, experiencias profesionales e investigaciones. Recuperado de file://C:/Users/Admin/Downloads/Dialnet-InteligenciaEmocionalYBienestarII-655308.pdf.

Espiñeira Bellón, E. (2018). Orientación a las familias en el periodo de adaptación. Revista de Estudios e Investigación en Psicología y Educación, n. ${ }^{\circ}$ 5, 273-277.

Esteban, A. (2015). Relación familia-escuela: Estudio descriptivo derivado de una experiencia de investigación-acción en el aula. Valladolid: Universidad de Valladolid.

García, J. L. (2003). Problemas y diseños de investigación resueltos. Madrid: Dykinson.

Garreta, J. (2015). La comunicación familia-escuela en Educación Infantil y Primaria. Revista de la Asociación de Sociología de la Educación, 8 (1), 71-85.

Hernández, M. ${ }^{a}$ A.; Gomariz, M. ${ }^{a}$. A. y Parra, F. (2015). El sentimiento de pertenencia en la relación entre familia y escuela. Madrid: Ministerio de Educación, Cultura y Deporte. 
PURIFICACIÓN CRUZ CRUZ Y MÓNICA PATRICIA BORJAS IMPORTANCIA DE LOS PRIMEROS PASOS EN LA RELACIÓN FAMILIA-COLEGIO. (DISEÑO DEL PERIODO DE ADAPTACIÓN EN EDUCACIÓN INFANTIL)

Julio, V.; Girón M. y Navarro, L. (2012). Estrategia educativa para la participación de los padres en compromisos escolares. Escenarios, 10 (2), 119-127. doi: 10.15665/esc.v10i2.226.

León, B. (2011). La relación familia-escuela y su repercusión en la autonomía y responsabilidad de los niños/as. En XII Congreso Internacional de Teoría de la Educación. Universidad de Barcelona. Recuperado de http://cort.as/-MQoN.

Martín, E. (2015). Escuela de padres y madres como mecanismo de prevención social. Tesis de Pregrado. Recuperado de http://xurl.es/t63ko.

Martín-Moreno, Q. (2007). Organización y Dirección de Centros Educativos Innovadores. El Centro Educativo Versátil. Madrid: Editorial McGraw-Hill.

Martín-Quintana, J.; Maiquez, M.; Rodrigo, M.; Byme, S.; Rodríguez, B. y Rodríguez, G. (2009). Programas de educación parental. Intervención Psicosocial. Revista Psychosocial Intervention, 18 (2), 121-133. Recuperado de: https://n9.cl/X6Hw.

Martínez González, R. A.; Rodríguez Ruiz, B. y Gimeno Esteo, J. L. (2010). Áreas de cooperación entre los centros docentes y las familias. Estudio de caso. Educatio Siglo XXI. Revista de la Facultad de Educación, 28 (1), 127-156. Recuperado de http://xurl.es/ffklt.

Ministerio de Educación, Cultura y Deporte (2014). La participación de las familias en la educación escolar. Madrid: Secretaría General Técnica.

Monarca, H. A. (2013). Trabajo colaborativo con padres y madres. Ámbito de actuación desde la orientación educativa. Revista Española de Orientación y Psicopedagogía, 24 (3), 114-123. Recuperado de http://xurl.es/qbk8r.

Montalbán, R. (2018). Infancia y educación Infantil. Recuperado de https://n9.cl/3jh.

Muñoz, J. M.; Espiñeira, E. M. y Losada, L. (2016). Planificación del periodo de adaptación en la Comunidad Autónoma de Galicia: revisión bibliográfica y legislativa. En J. R. Casanova; C. Bisinoto y L. S. Almeida (Eds.). IV Seminário Internacional Cognição, Aprendizagem e Educação (pp. 200-211). Braga, Portugal: Centro de Investigação en Educação (CIEd).

OCDE (2012). Starting Strong III - A Quality Toolbox for Early Childhood Education and Care. Paris: OCDE.

Páez, R. M. (2015). Familia y escuela: una alianza necesaria en la gestión escolar. Revista Iberoamericana de Educación, 67, 159-180.

Pérez, C. (2011). Los primeros días de clase en Educación Infantil. Pedagogía Magna, 9, 89-94. Recuperado de file://C:/Users/Admin/Downloads/Dialnet-LosPrimerosDiasDeClaseEnEducacionInfantil-3628215.pdf.

Petrakos, H. T. y Lehrer, J. S. (2011). Parents' and teachers' perceptions on transition practices in kindergarten. Exceptionality Education International, 21 (2), 62-73. Montreal: Concordia University.

Poole, A.; Miller, D. y Booth, E. (2019). Ages \& Stages: How Children Adjust to School. Recuperado de http://cort.as/-MQna.

Quintero, M. (2016). El papel de la familia en la Educación. Revista digital. Investigación y Educación, n. ${ }^{\circ}$ 21, 1-7. Recuperado de https://n9.cl/cG2U.

Rodríguez Fernández, S. y Romero Lozano, M. (2015). La función tutorial en educación infantil y primaria: desempeño profesional del profesorado. Revista Electrónica Interuniversitaria de Formación del Profsorado, 18 (2), 43-55. Recuperado de http://xurl.es/vd6lj. 
PURIFICACIÓN CRUZ CRUZ Y MÓNICA PATRICIA BORJAS

Sánchez, E. (2007). El periodo de adaptación a la escuela Infantil. Granada: Editorial de la Universidad de Granada.

Soler, A. (2018). Adaptación a la escuela. Claves para ayudar a los peques. Recuperado de https://n9.cl/3Yk2.

United Nations Children's Fund School. UNICEF (2012). Readiness: a conceptual framework. New York: United Nations Children's Fund Education Section.

Vallespir, J.; Rincón, J. C. y Morey, M. (2016). La participación de las familias en el Consejo Escolar y la formación del profesorado. Revista Electrónica Interuniversitaria de Formación del Profesorado, 19 (1), 31-45.

Valles, M. (2014). El periodo de adaptación en el segundo ciclo de Educación Infantil. Tesis doctoral. Universidad Internacional de la Rioja. Recuperado de http://xurl.es/lr1jx.

Vila, I. (2014). Familia y escuela: dos contextos educativos y un solo niño. Barcelona: Grao Editorial.

Zabalza, M. Á. (2015). La educación infantil como tarea compartida. Reladei, 4 (2), julio 2015, 13-15. ISSN: 2255-0666. 